\title{
Cartographie descriptive du système national français du financement de la recherche sur projet en vue de son évaluation
}

\author{
Romain TOURET \\ Université Paris-Dauphine \\ PSL Research University, CNRS, LAMSADE \\ romain.touret@dauphine.fr \\ Yves MEINARD \\ Université Paris-Dauphine \\ PSL Research University, CNRS, LAMSADE \\ yves.meinard@dauphine.fr
}

Jean-Claude PETIT

Université Paris-Dauphine PSL Research University, Présidence jean-claude.petit@dauphine.fr

Alexis TSOUKIAS

Université Paris-Dauphine PSL Research University, CNRS, LAMSADE RÉSUMÉ

alexis.tsoukias@dauphine.fr

Dans cet article nous proposons une cartographie du système national du financement de la recherche (SNFRI) français. Cette représentation s'appuie sur un modèle fonctionnel et organisationnel du système que nous faisons évoluer à travers l'ajout d'une fonction Évaluation. Cette dernière est conçue avec l'ambition de fournir une analyse de l'impact du système de recherche dans sa globalité plutôt qu'un examen qualitatif des projets et des acteurs qui le composent. Cette carte est enfin une attestation empirique de l'existence et de la pertinence de ces fonctions et constitue la première étape d'un travail plus large dont l'objectif final est de construire un modèle de SNFRI capable de l'évaluer et d'appréhender la dynamique évolutive globale du financement de la recherche en France.

MOTS-CLÉS : Financement de la recherche, Système National d'Innovation, Policy Analytics, Évaluation, Impact de la R\&D, Aide à la décision

CODES JEL : O38, 123, 128, H11, H52 


\section{ABSTRACT \\ Descriptive Mapping of the French National System of Financing Project Research for Its Évaluation}

In this article, we propose a novel cartography of the French national research funding system (SNFRI). This representation is based on a functional and organizational model of the system that we modify through the addition of an evaluation function. The latter is designed with the ambition to provide a synthetic analysis of the impact of the research system as a whole rather than a qualitative examination of the projects and the actors that compose it. Finally, this map is an empirical validation of the existence and relevance of these functions and constitutes the first stage of a broader work whose ultimate objective is to build a model of SNFRI able to evaluate it and to grasp the global evolution dynamics of the financing of research in France.

KEYWORDS: Research Funding, National Innovation System, Policy Analytics, Évaluation, Impact of $R \& D$, Decision Support

JEL CODES: O38, 123, 128, H11, H52

Cet article s'intéresse à la dynamique d'évolution d'un système de financement de la recherche. Nous cherchons à comprendre l'ensemble formé par le système de financement de la recherche et la politique dans laquelle il s'inscrit comme un ensemble dynamique, dont les caractéristiques évoluent, entre autres en réponse à des ajustements de structure qu'une méthode d'évaluation du système satisfaisante serait capable de préconiser.

Plus précisément, les questions qui vont nous occuper sont les suivantes : Dans le cadre d'une politique de recherche donnée, quelle méthode d'évaluation du système de financement de la recherche faut-il déployer pour faire en sorte que les projets financés permettent d'atteindre les objectifs fixés dans le cadre de cette politique? Et, s'ils ne le permettent pas, comment alerter les décideurs que le système s'oriente dans une direction différente de celle escomptée?

Trois principaux types d'approches peuvent être mis à contribution pour répondre à ces questions :

1. Une approche historique (Freeman, 1995), qui relate l'évolution factuelle des systèmes considérés ;

2. Une approche comportementale (Lundvall, Jonhson, 1994), qui étudie la manière dont les acteurs se positionnent dans le système notamment en matière d'apprentissage, les motivations qui les animent et les incitations auxquels ils répondent ;

3. Une approche modélisatrice ${ }^{1}$, qui s'articule autour de l'identification et de la modélisation des différentes fonctions qui constituent le système,

1. La notion de modèle est comprise ici en son sens usuel en sciences sociales, comme une représentation simplifiée et compréhensible d'une situation réelle plus complexe (Schneider, 1996, parle du principe de similarité) réalisée pour comprendre ou expliquer un phénomène précis (principe d'utilité de Schneider). 
dans la lignée des travaux sur les systèmes nationaux d'innovation (SNI)

(Nelson, 1993) et leur dynamique.

C'est dans cette dernière dynamique que nous nous inscrivons dans ce travail, en développant un modèle du système de financement de la recherche.

Lapproche historique appliquée au système français a été développée par (Heraud, Lachmann, 2015). L'Annexe rappelle les éléments les plus pertinents de cette analyse de notre point de vue. Lapproche comportementale n'a, à notre connaissance, pas encore été déployée pour la France. Il est à souhaiter qu'elle le soit car les résultats de son application pourraient être complémentaires à la fois avec nos résultats et avec ceux de l'approche historique.

Dans le cadre de l'approche de modélisation des fonctions qui est la nôtre, nous proposons de cartographier le système de recherche et d'innovation français en nous intéressant tout particulièrement au financement de la recherche, selon une méthodologie d'observation participante opportuniste (Soulé, 2007).

En déployant cette démarche, nous montrerons que, bien que les trois fonctions proposées par (Barré, 2007 ; Barré, 2011) (Orientation, Programmation et Recherche) soient présentes dans le système de financement de la recherche en France, il convient de leur ajouter une quatrième fonction, l'Évaluation, qui rend compte d'une remontée de l'information responsable de la dynamique du système.

Cet article est organisé comme suit. Dans une première partie, nous présentons les grilles de lecture des SNI présentes dans la littérature et mettons en évidence l'originalité de notre apport. Une deuxième partie est alors consacrée à l'explication de notre méthodologie. Enfin, nos résultats (la cartographie du SNFRI) sont présentés dans une troisième partie.

\section{Les analyses des systèmes nationaux d’innovation}

\section{La notion de « systèmes nationaux d'innovation »}

Le concept de système d'innovation (SI) est le concept-clef des travaux de recherche qui visent à incorporer des éléments institutionnels dans l'analyse économique du changement technique (Amable, 2002). Le point de départ des travaux sur les SI est un changement de paradigme dans la conception de l'innovation, qui n'est plus vue comme un processus de décision individuel indépendant de l'environnement, mais comme le fruit du fonctionnement d'un réseau d'acteurs insérés dans différentes institutions (Casadella, BenlahcenTlemcani, 2006). L'innovation est vue comme le résultat d'interactions entre 
ces acteurs (entreprises, laboratoires, universités...) et leur environnement, régies par un ensemble de règles et d'organisations.

Le SNI (système national d'innovation) ou SNRI (système national de recherche et d'innovation) est un concept relativement récent introduit dans les années 1980 (Freeman, 1987 ; Lundvall, 1992 ; Nelson, 1993 ; Edquist, 1997) pour expliquer les interactions entre public, privé, universités et agences dans la production nationale de science et technologie (Lundvall et al., 2002 ; Edquist, 2006 ; Lundvall, 2007). Dans ce cadre, les études des systèmes sectoriels d'innovation (SSI ; Malerba, 1999 et 2002) et des systèmes d'innovation technologique (SIT ; Carlsson et Stankiewicz, 1995 ; Hughes, 1990) limitent l'analyse respectivement à un secteur ou à une technologie.

Le périmètre de telles études peut être plus ou moins étendu et dépend du nombre d'interactions considérées entre agents du système (Amable, 2002). Se pose ainsi le problème de la délimitation du système. La Figure 1, inspirée de Hekkert et al. (2007), présente les frontières des SNI, des SSI et des SIT. Dans cet article nous étudions un système à un niveau national : nous nous plaçons donc dans le contexte des SNI.

Figure 1 - Relations entre les frontières nationales, sectorielles et technologiques des systèmes d'innovation

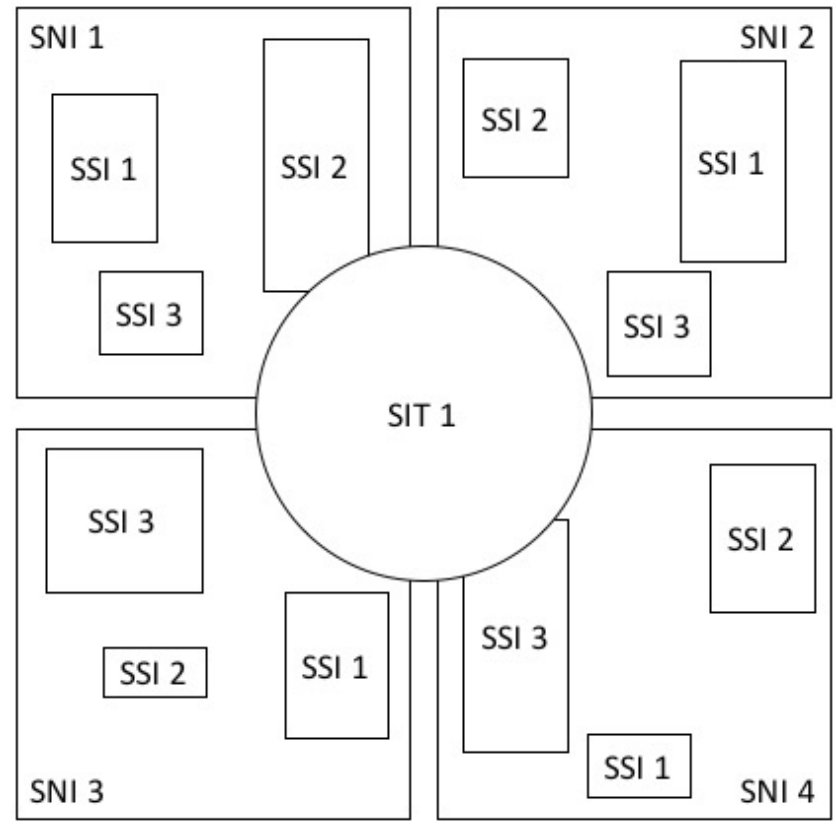

Source : inspiré de Hekkert et al (2007) - SNI = systèmes d'innovation national, SSI = systèmes sectoriels d'innovation et SIT = systèmes d'innovation technologique. 
Bien que "[l]a définition du système [soit] la plupart du temps floue " (Amable, 2002, p. 5), quelques études tentent de la préciser notamment à travers la définition des fonctions du SI. La Figure 2 schématise ce processus.

Figure 2 - Définition du périmètre d'un système à travers sa modélisation fonctionnelle
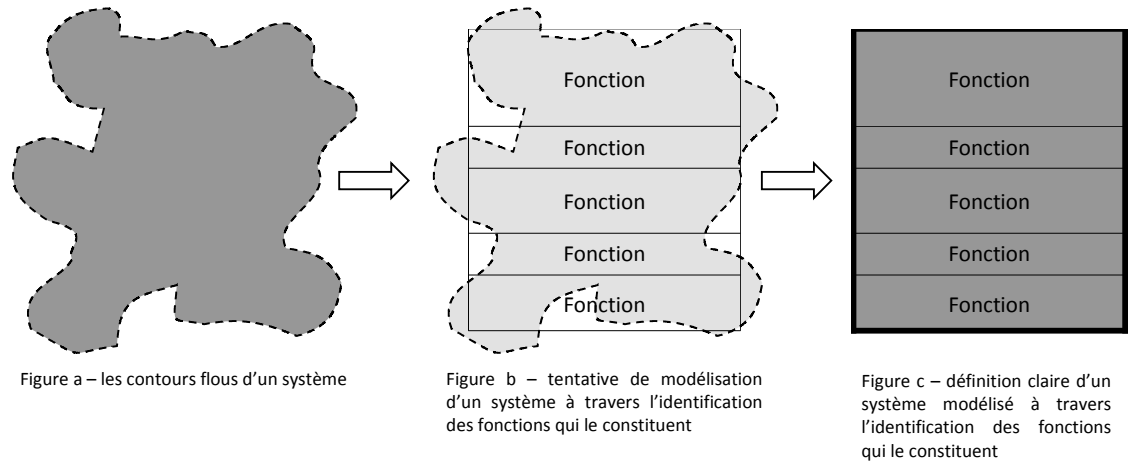

Sur ce front, les travaux des équipes de Chalmers (Rickne, 2001 ; Johnson, 2001 ; Bergek, Jacobsson, 2003 ; Jacobsson et al., 2004 ; Bergek et al., 2008), et d'Utrecht (Negro et al., 2007 ; Hekkert et al., 2007 ; Hekkert, Negro, 2009) sont arrivées à des listes de fonctions équivalentes.

La formalisation de fonctions dans le SNRI permet :

1. De définir les frontières du système : le SI contient toutes les composantes qui influencent une ou plusieurs fonction(s) identifiée(s) (Johnson, 2001, p. 16).

2. De décrire l'état d'un système à travers l'identification des mécanismes qui peuvent induire un blocage de certaines fonctions.

3. D'étudier la dynamique du système d'innovation : « La cartographie du motif fonctionnel » (traduit de l'anglais, Johnson, 2001, p. 16), c'est-à-dire l'étude de la manière dont les fonctions ont été remplies au fil du temps, donne une image de la manière dont le système a émergé.

4. D'évaluer la performance du système en examinant dans quelle mesure les fonctions ont été remplies (Johnson, 2001, p. 17).

Bien que les SI aient été étudiés à un niveau national, il n'existe à notre connaissance aucune étude de leur dynamique d'évolution à cette échelle, et donc aucune recherche sur les fonctions du système à ce niveau. Limmense majorité des travaux décrivent un système actuel, sans s'intéresser ni aux forces qui l'ont construit et le font évoluer, ni à son organisation future. Selon Hekkert et al. (2007), ceci s'explique par une très grande difficulté à cartographier cette dynamique dans son ensemble, en particulier à cause de l'extrême 
complexité des systèmes due au nombre trop important d'acteurs, de relations et d'institutions.

Avec l'émergence d'études focalisées sur les systèmes d'innovation technologique (SIT), le nombre d'acteurs, de réseaux et d'institutions considérés (pour la technologie étudiée) s'est considérablement réduit du fait d'une limitation du périmètre de l'objet d'étude (on exclut du SIT tous les éléments qui ne prennent pas part au développement de la technologie étudiée). Avec les études de SIT, on diminue ainsi la complexité du système tout en rendant possible l'étude de sa dynamique à travers des schémas d'analyse basés sur l'identification de ses fonctions (Hekkert et al., 2007 ; Bergek et al., 2008). D'après Hekkert et Negro (2009), cette approche n'est cependant pas encore aboutie et le modèle théorique doit encore être stabilisé.

Nous proposons ici une approche de SNI qui se concentre uniquement sur le financement de la recherche sur projet, de façon à limiter la complexité du système. Nous nommons l'objet ainsi circonscrit : «système national du financement de la recherche et de l'innovation » (SNFRI). Utilisant le même raisonnement que les études de SIT, nous cherchons à identifier les fonctions du SNRFI, dans l'optique d'étudier sa dynamique.

Figure 3 - Ressources de la recherche publique
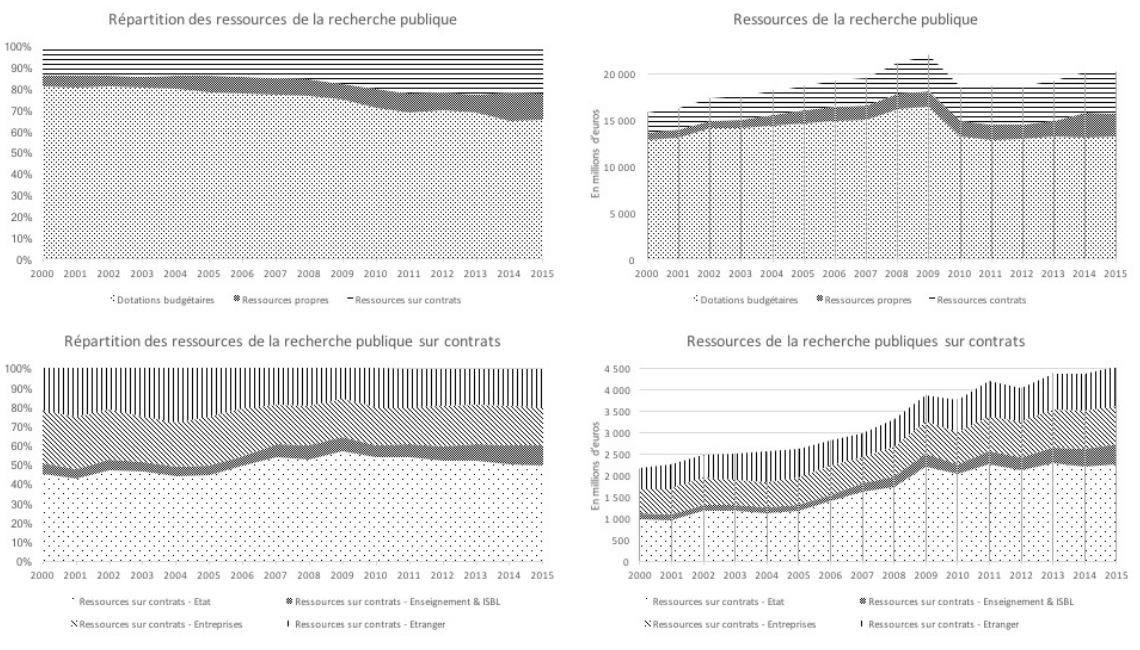

Source : MENESR-DGESIP/DGRI-SIES

Cette limitation au SNFRI peut de prime abord sembler réductrice. Cependant, dans le système français, le financement sur projet tend de fait à prendre de plus en plus d'importance au détriment des dotations globales. La Figure 3 illustre cette évolution (absolue et relative) des ressources de la 
recherche publique française depuis $2000^{2}$. On constate une diminution des dotations budgétaires au profit des ressources sur contrat (financement sur projet) et, dans une moindre mesure, des ressources propres (redevances de la propriété intellectuelle, dons, legs et prestations de services). La limitation au SNFRI n'est, en ce sens, pas une restriction aussi drastique qu'il n'y paraît. Ceci étant, cette limitation a inévitablement un impact sur la portée de notre étude, qu'il conviendra de garder à l'esprit au moment d'en tirer des enseignements.

\section{L'étude du SFRI : théorie fonctionnelle et organisationnelle}

Barré (Barré, 2007 ; Barré, 2011) a construit une représentation fonctionnelle et organisationnelle du système français de recherche et d'innovation (SFRI). Ses travaux font apparaître différentes fonctions portées par des acteurs en interactions, qui sont tous porteurs d'une «intelligence stratégique ».

Lauteur définit ainsi trois fonctions du SFRI :

- La fonction Orientation: portée par le gouvernement, elle consiste à définir une vision du devenir du système et des objectifs généraux, ainsi qu'un volume de dépenses consacré à la $R \& D$. Elle relève de la responsabilité du politique à différentes échelles (locale, nationale, communautaire) ;

- La fonction Programmation : portée par les agences publiques de financement de la recherche, cette fonction consiste, d'une part, à traduire les orientations du gouvernement en priorités programmatiques et thématiques et, d'autre part, à affecter des ressources à des opérateurs de recherche. Elle relève de la responsabilité des organismes de recherche, des agences de financement et des directions de recherche des ministères ;

- La fonction Recherche: portée par les opérateurs de recherche, elle consiste en la réalisation concrète de la recherche. Elle relève de la responsabilité des institutions de recherche et des établissements d'enseignement supérieur.

Pour Barré, «le modèle auquel renvoie ce référentiel est celui d'un fonctionnement interactif entre les acteurs situés au sein de chacune des fonctions, mixant les démarches 'bottom-up' et 'top-down', en proportions variables selon les domaines et types de recherche » (Barré, 2011, p. 10). Ceci est d'autant plus vrai que même si les fonctions sont bien distinctes, les mêmes personnes

2. On peut noter une cassure de la série entre 2009 et 2010. Celle-ci s'explique par un changement méthodologique dans la prise en compte de certaines ressources. 
(généralement des chercheurs) peuvent être impliquées simultanément dans deux, voire trois fonctions.

Les limites entre fonctions sont donc perméables, autant en termes de réalisation opérationnelle que de localisation des compétences. La ressource humaine de chaque fonction est fournie pour l'essentiel par la fonction Recherche. À travers leur présence généralisée dans tout le système, les opérateurs issus de cette dernière construisent ainsi le point de vue de l'Orientation et de la Programmation qui en résulte.

De ce fait, pour Barré, la description complète du SFRI passe par la description des interactions entre chaque fonction, puis par l'observation des sous-systèmes communautaires, régionaux et industriels (ces sous-systèmes assument chacun à leur niveau une, deux, ou trois fonctions) et, enfin, par la caractérisation des relations entre ces sous-systèmes et les institutions nationales.

L'organisation du SFRI est enfin caractérisée par la mise en œuvre de ses fonctions. Barré définit ainsi deux types d'organisation :

- Le modèle séparé où une institution (et un homme) ne peut assumer qu'une et une seule fonction ;

- Le modèle intégré dans lequel une institution peut assumer plusieurs, voire toutes les fonctions.

Aucun système réel n'est totalement intégré ou totalement séparé. Barré parle ainsi de modèle majoritairement intégré ou séparé.

\section{L’organisation séparée}

Cette organisation se résume à une succession de relations hiérarchiques :

1. Le gouvernement fixe les orientations, les objectifs et le cadre financier par grand domaine de recherche.

2. Les agences publiques font la traduction des orientations en objectifs scientifiques, en développement de compétences, d'infrastructures et de partenariats. Elles allouent les ressources aux opérateurs de recherche.

3. Les opérateurs de recherche utilisent les fonds obtenus pour réaliser des travaux de recherche.

Cette hiérarchie entre acteurs séparés est observable notamment au niveau de la transmission des objectifs de la politique qui suit une direction de haut en bas, de l'Orientation à la Recherche en passant par la Programmation. Ainsi, l'orientation donnée par le gouvernement est traduite par les agences publiques avant d'être théoriquement suivie par les opérateurs de recherche. Cette information est en revanche dégradée ou érodée à mesure qu'elle descend dans le système, dans le sens où elle est traduite, retraduite et transmise 
à plusieurs reprises avant d'arriver à la Recherche, si bien que l'orientation initiale est généralement très déformée lorsqu'elle a atteint le bas du système. Ce phénomène d'érosion implique parfois des difficultés pour comprendre le financement d'un projet très éloigné de la direction donnée initialement par le gouvernement. Ceci est d'autant plus visible que l'appel à projets est ancien et dispose de sa propre jurisprudence impliquant une inertie difficilement compatible avec une réelle réactivité à l'orientation.

Pour Barré, un modèle séparé présente les avantages suivants :

- Efficacité de la fonction recherche à travers la valorisation de la recherche universitaire, la prise en compte de l'ensemble des compétences du système, tout en facilitant l'interdisciplinarité et l'implication des opérateurs de recherche.

- Lisibilité relative du SNRI, avec notamment la possibilité de faire le lien entre l'Orientation, la Programmation et l'allocation des ressources budgétaires.

- Possibilité d'évaluer les parties prenantes du système selon la fonction exercée.

Pour Barré, le modèle séparé n'est pour autant pas exempt de défauts. Il repose sur une formalisation importante des liens entre les différentes fonctions et la définition d'un périmètre d'intervention précis pour l'ensemble des parties prenantes du système. Ce formalisme engendre des coûts qui peuvent être importants, limitant ainsi le gain attendu de cette organisation. Enfin, il suppose une réactivité importante de l'ensemble des fonctions pour ne pas risquer de voir un dysfonctionnement se propager à l'ensemble du système.

\section{L'organisation intégrée}

Cette organisation est caractérisée par des instituts de recherche nonuniversitaires et thématiques, ayant tous pouvoirs ou presque, réalisant pour eux-mêmes la Programmation voire dans certain cas l'Orientation. Cette organisation présente l'intérêt d'être plus simple à mettre en œuvre puisqu'il suffit de réunir, pour un domaine donné, l'ensemble de la recherche dans un institut de recherche indépendant. Cette organisation est très efficace lorsque l'on connaît par avance la direction scientifique à suivre, dans un contexte de rattrapage technologique par exemple. Enfin, l'organisation intégrée jouit d'une relative robustesse, dans le sens où sa construction en silos indépendants permet de circonscrire un événement à un silo sans que celui-ci ne contamine l'ensemble du système. Ce phénomène de silo permet en outre aux opérateurs de recherche de prendre des risques en explorant de nouveaux domaines, sans craindre d'impacter négativement l'ensemble du système. 
En revanche, cette organisation ne permet pas de synergie entre les domaines de la recherche et limite la connaissance nationale de la thématique à celle produite par son institution. Le poids des fonctions supérieures ferme ainsi la porte aux travaux collaboratifs nécessaires dans certains domaines (voir par exemple Letourneau, 2008). Enfin, le modèle à fonctions intégrées reste très opaque pour le grand public, voire pour le gouvernement, qui fait difficilement le lien entre les allocations budgétaires et les résultats scientifiques produits. Ceci s'explique en particulier par l'intégration de toutes les fonctions au sein d'une même institution rendant difficile pour le citoyen la lisibilité de la structure stratégique de la politique de recherche.

\section{L'évolution d'une organisation intégrée vers une organisation séparée via un stade hybride}

Si l'on lit l'histoire du SFRI de 1945 à nos jours (voir notamment Heraud et Lachmann (2015) et Mustar et Larédo (2002), qui décrivent, au tournant des années 2000, la fin du modèle Colbertiste instauré à la fin de la seconde guerre mondiale - détaillé en Annexe) à la lumière des modèles présentés précédemment, on comprend que le système français s'est initialement construit selon une organisation intégrée avant de progressivement évoluer vers un modèle séparé.

Pour Barré, "La loi sur la recherche de 1982 constitue l'expression aboutie du schéma à 'fonctions majoritairement intégrées'. De ce point de vue, elle peut être considérée comme l'accomplissement des évolutions qui ont marqué la période d'après-guerre, caractérisée par la création de ces organismes intégrés dans une optique de rattrapage volontariste » (Barré, 2007, p. 12). Ce modèle n'est cependant pas compatible avec la compétition mondiale en matière de recherche et d'innovation apparue au tournant du $21^{\mathrm{e}}$ siècle. L'ensemble des observateurs et des indicateurs de performance de cette époque montrent que le SFRI est à la traîne sur la scène internationale, et ce malgré les efforts décrits précédemment. Le sentiment dominant de cette époque est celui d'une certaine impuissance, une partie importante de l'énergie étant consacrée à contourner les obstacles administratifs.

La loi de programme de la recherche de 2006 cherche à répondre à cette inquiétude en créant des institutions et des instruments permettant de faire évoluer le modèle vers une organisation séparée avec pour objectifs de :

- Construire une fonction Orientation plus forte.

- Rationaliser la fonction Programmation avec notamment la création de l'ANR.

- Mettre en valeur la fonction Recherche et faire émerger des opérateurs différenciés.

- Permettre une évaluation systématique des parties prenantes. 
Ainsi, depuis plus de 10 ans, l'Orientation cherche à séparer un système qui s'était construit sur un modèle très largement intégré. En faisant basculer le système vers une organisation majoritairement séparée, la réforme de 2006, confortée par la loi de 2013, est un tournant majeur de l'organisation de la recherche en France. L'histoire du SFRI reste cependant très marquée par le modèle intégré, comme en atteste la recherche qui est menée par de grandes institutions telles que le CEA et le CNRS, où l'Orientation et la Programmation restent encore très largement intégrées (ou trop selon les points de vue).

Le SFRI se présente ainsi comme une synthèse entre une histoire très marquée par une organisation intégrée et une volonté affichée des politiques de la séparer, résultant en un modèle que l’on peut appeler « hybride ».

Le modèle de Barré fournit donc une grille de lecture éclairante, mais il ne rend pas compte de l'existence de mécanismes permettant à l'Orientation de faire remonter l'information sur l'atteinte de ses objectifs, contrairement, par exemple, aux travaux de Carlsson et al. (2002) décrivant la construction d'un système d'innovation à travers un mécanisme de retour d'information (Feedback). Cette lacune dans le modèle de Barré le rend incapable d'appréhender la dynamique du système.

\section{Dépasser les modèles actuels pour comprendre la dynamique du système}

Notre objet dans cette étude est de dépasser la limite identifiée dans le modèle de Barré. Pour cela, nous montrerons qu'il convient d'ajouter aux trois fonctions de Barré une fonction Évaluation, dont l'objet est de faire remonter l'information dans le système. En effet, alors que Barré intègre l'Évaluation dans l'Orientation, faire de l'Évaluation une fonction à part entière permet de mettre en exergue l'influence des démarches d'évaluation sur la décision et plus particulièrement sur les choix politiques (Marchant et al., 2003 ; Marchi et al., 2014 ; Bouyssou et al., 2000), et ainsi d'expliquer les choix politiques réalisés par l'Orientation.

\section{Méthodologie pour la cartographie}

\section{L'observation participante comme cadre méthodologique}

Pour cette étude, nous avons mis en œuvre une méthodologie qui a consisté à réaliser un travail long et approfondi de consulting en montage de projets de recherche pour la capture de financements, et à abstraire de cette pratique 
de consulting les éléments-clefs du modèle de fonctionnement du système. Cette méthodologie qui abstrait les éléments de connaissance à partir de la pratique performative du consulting s'inscrit dans la lignée de la méthode, plus classique en sciences sociales, d'observation participante.

Lobservation participante consiste à réaliser une étude en «immersion totale dans son terrain » (Soulé, 2007, p. 2). Cette méthode, apparue dans les années 1930 (Platt, 1983), vise à permettre aux chercheurs de vivre la réalité des sujets qu'ils observent. Malgré son ancienneté, elle n'a encore jamais été complètement formalisée. Elle recouvre en conséquence une multitude de techniques d'observation, qui ont toutes en commun l'immersion de l'observateur dans son terrain d'étude.

Soulé (2007) distingue observation participante ouverte et clandestine (Abercrombie et al., 2000 utilisent le terme de « couverte ») selon que le chercheur se présente comme tel ou pas devant les acteurs. L'opération sous couverture permet de saisir les processus sociaux dans leur contexte « naturel », y compris dans des groupes généralement opposés à toute observation extérieure (De Sardan, 2001 ; Diaz, 2005). L'observation clandestine pose cependant des problèmes éthiques et les chercheurs préfèrent généralement se déclarer.

Les différentes formes d'observation participante se distinguent également par le degré de participation du chercheur (De Sardan, 2001). La question est " [c]omment concilier la nécessité méthodologique de l'implication dans la vie d'un groupe avec le recul et la mise en perspective nécessaires au rôle du chercheur » (Diaz, 2005). Ceci constitue la principale critique opposée à cette technique, certains sociologues soulignant notamment le risque de manque de d'objectivité de l'analyste lorsqu'il est partie prenante du jeu social. Adler et Adler (1987), préconisent en conséquence une observation participante "périphérique », dans laquelle la participation du chercheur est alors plus modérée, afin de ne pas être «aspiré » par l'action. Hughes (1996) parle «d'émancipation » pour qualifier la démarche qui consiste à trouver un équilibre entre le détachement et la participation.

Enfin, Soulé (2007), distingue l'observation participante opportuniste, où une personne opère une conversion de simple partie prenante au groupe à analyste, de l'observation entriste, où le chercheur s'immerge volontairement dans son terrain dans un objectif scientifique.

Dans notre cas, notre objectif était davantage la compréhension de la place de chaque organisation dans le système que l'observation des liens sociaux entre acteurs. Notre immersion dans le système est de ce point de vue moins problématique qu'elle ne peut l'être dans certains autres types d'études.

Dans les termes de ces débats méthodologiques, notre travail relève de la participation observante opportuniste, non périphérique et ouverte. 


\section{Concrétisation de la méthodologie et de son application}

Notre travail s'est déployé au sein un cabinet de conseil dont la mission est d'assister des porteurs de projets dans le financement de leurs travaux.

Les missions qui nous ont servi de support concernent des projets pour lesquels la demande de financement a pu être conduite jusqu'à son terme ainsi que des projets qui n'ont pas passé cette étape. En près de 7 ans, nous avons ainsi contribué au financement d'en moyenne 10 projets de recherche par an, auxquels s'ajoute le même nombre de projets avortés. Nous nous appuyons donc ici sur l'étude de 150 projets. Ces derniers sont par ailleurs le reflet de la très grande diversité de la recherche française et de ses sources de financement sur projet. Nous avons ainsi accompagné des projets de tous types, nationaux, internationaux, collaboratifs, individuels, courts (moins de 36 mois), longs (plus de 36 mois)... De même, nous sommes intervenus sur des sujets aussi variés que l'économétrie, la finance, la chimie, l'énergie ou bien les sciences du langage.

Nous avons par ailleurs complété cette expérience par plusieurs dizaines d'entretiens avec différents acteurs publics, dont la très grande majorité a souhaité rester anonyme. Enfin, nous avons bénéficié de la pratique des équipes du cabinet pour la compréhension des différents dispositifs. Aussi bien les équipes de notre financeur que les différentes personnes interrogées étaient informées du contexte dans lequel nous les questionnions. En ce sens nous nous plaçons dans le cadre d'une observation participante ouverte.

Pour chacun des 150 projets de notre base d'étude, la première étape de l'accompagnement a toujours consisté à identifier les dispositifs à même de les financer. Cette recherche s'effectuant à travers un réseau de partenaires publics créé au fil des années, mais également à travers une recherche ciblée de dispositifs en fonction des caractéristiques de chaque projet. Parmi les critères les plus importants, citons la taille du projet (nombre de partenaires impliqués), sa durée, sa localisation géographique, les thématiques abordées, etc. À partir de là, on cible les organisations jugées les plus à même de financer (pour le dire rapidement, on contacte en priorité le Conseil Régional pour un projet à portée limitée, l'ANR - Agence nationale de la recherche - pour un projet de recherche fondamentale et l'ADEME - Agence de l'environnement et de la maîtrise de l'énergie - pour un projet en lien avec l'environnement ou l'énergie). En fonction des retours de ces financeurs potentiels, il est possible d'obtenir une liste assez réduite des dispositifs qui présentent la probabilité la plus importante de financer un projet. Enfin, nous nous intéressons aux projets déjà financés par ces dispositifs (ou par des dispositifs similaires dans le cas d'un nouveau programme) pour comprendre leurs critères de sélection. 
Pour réaliser la cartographie, nous avons utilisé ce travail en cherchant à comprendre le parcours des fonds en partant des projets financés et en essayant de remonter le plus haut possible dans le système. Plus précisément, pour chaque projet financé, une convention est établie entre le porteur de projet et le financeur ayant rédigé l'appel à projets. Ces documents précisent les modalités de l'aide et leur cadre juridique. En effet, les financements s'inscrivent dans des budgets qui eux-mêmes émanent d'autres budgets et ainsi de suite, si bien qu'à travers ce mécanisme d'analyse récursive, il est possible de reconstituer le parcours des fonds.

Nous avons enfin complété ce travail à la lumière des représentations simplifiées déjà existantes. En particulier, une première ébauche d'une organisation fonctionnelle du système a été réalisée par le ministère de la recherche ${ }^{3}$. Weisenburger et al. (2014) proposent quant à eux l'introduction d'un processus vertical hiérarchisé ainsi qu'une distinction entre entités qui financent et réalisent la $R \& D$. Cette séparation fait écho aux agrégats statistiques de la DNRD et de la DIRD ${ }^{4}$. Nous reviendrons plus loin sur la difficulté de cette distinction, en particulier lorsqu'une même entité finance et exécute la $R \& D$ ou lorsqu'elle n'est amenée qu'à instruire une demande de financement sans prendre part à la décision ou aux travaux futurs de recherche.

\section{Limites de la méthodologie}

Nous avons défendu dans les paragraphes précédents l'intérêt d'une approche par observation participante pour l'étude de notre objet. Reconnaissons cependant qu'une limite de notre étude est sa non-exhaustivité. En effet, établir une cartographie comme celle qui nous intéresse suppose de balayer de manière quasiment exhaustive les dispositifs de financement pertinents. Cependant, si nous avions voulu garantir l'exhaustivité de notre travail, il aurait été nécessaire de passer par un recensement de l'ensemble des dispositifs du financement de la recherche pour ensuite en déduire un schéma général. Un tel exercice s'avère en pratique impossible tant ces dispositifs sont nombreux ${ }^{5}$,

3. http://www.enseignementsup-recherche.gouv.fr/cid56382/organisation-du-systeme-de-recherche-etd-innovation.html

4. En France, la Cour des Compte (Cour des comptes, 2013) appréhende l'effort de recherche et développement par un agrégat principal de comptabilité nationale, la dépense intérieure de recherche et développement (DIRD). Celle-ci se subdivise en DIRD des administrations (la DIRDA) et DIRD des entreprises (la DIRDE). Elle obéit à une définition méthodologique commune aux pays membres de l'OCDE, contenue dans le Manuel de Frascati (OECD, 2002). Elle est complétée par un autre agrégat, la dépense nationale de recherche et développement (la DNRD), qui permet de mesurer la dépense de recherche financée sur des ressources publiques ou privées françaises, qu'elle soit exécutée en France ou à l'étranger. Ainsi, la DNRD correspond au financement de la recherche tandis que la DIRD reflète son exécution.

5. La recherche peut être financée localement, régionalement, nationalement et communautairement. De ce fait, à notre connaissance personne n'a jamais entrepris de recenser tous ces dispositifs tant il est presque impossible de les identifier tous. Une personne qui a souhaité garder l'anonymat nous a confirmé cette 
d'autant que presque chaque jour des dispositifs sont abandonnés et d'autres sont créés.

Notons enfin, au titre des limites, que, pour une meilleure compréhension, nous avons fait le choix de réduire le périmètre de notre étude à l'écosystème qui gravite autour de la mission interministérielle recherche et développement (MIRES) ${ }^{6}$. Ce choix induit inévitablement une perte d'exhaustivité. Néanmoins, comme la MIRES représente l'essentiel du budget de la recherche en France, il paraît réaliste d'affirmer que cette limitation n'a qu'un effet mineur sur la validité de l'étude.

Enfin, insistons sur le fait que cette étude n'a pas de prétentions empiriques quantitatives. En revanche, elle pourrait servir de base à un travail statistique ou méta-analytique.

\section{Construction de la carte du SNFRI français}

Dans ce qui suit, nous proposons de construire notre carte du système français de financement de la recherche. Pour cela, nous commençons par introduire et justifier l'introduction d'une quatrième fonction, l'Évaluation, qui vient compléter le modèle de Barré. Nous détaillons alors chacune de ces fonctions à travers les liens qui les unissent et le rôle qu'elles jouent dans le processus de décision de financer un projet. Une fois ce travail terminé, nous chercherons à expliquer, uniquement en utilisant cette carte, comment passer de la définition d'une politique publique au financement d'un projet de recherche. Si nous y parvenons, nous aurons ainsi validé la pertinence de ces fonctions.

\section{La fonction Évaluation}

Nous ajoutons à l'organisation proposée par Barré une quatrième fonction :

- La fonction Évaluation : portée principalement par un organisme indépendant dédié, cette fonction est investie par l'Orientation d'une mission

difficulté à travers l'exemple de la Cours de Comptes qui bien qu'elle dispose d'un droit de regard autrement plus important que celui d'un simple chercheur, découvre très régulièrement des dispositifs dont elle n'avait pas encore connaissance.

6. Depuis 2006 et l'entrée en vigueur totale de la loi organique relative aux lois de finance (LOLF) qui instaure un nouveau paradigme budgétaire centré autour d'une logique de performance d'une part et de transparence d'autre part, le budget dispose d'objectifs clairement indiqués et d'indicateurs chiffrés pour valider l'atteinte de ces objectifs. En outre, la LOLF instaure une segmentation du budget en 34 missions interministérielles, 132 programmes et 580 actions qui détaillent les finalités des politiques publiques. C'est dans ce contexte qu'a été créée la MIRES qui est composée de 10 programmes dont 5 relèvent du ministère de la recherche. Ces derniers regroupent près de $90 \%$ des crédits de la mission. À lui seul, le programme « Formations supérieures et recherche universitaire » représente près de la moitié des crédits de la mission. 
d'information. Sa responsabilité est l'évaluation du système, et en particulier des opérateurs de recherche et des agences de financement.

La fonction Évaluation n'est en aucun cas en charge de l'expertise projet. En d'autres mots : son rôle n'est pas de dire si un projet est bon ou mauvais. Décrite de manière informelle, l'Évaluation est une fonction qui agrège l'impact de tous les projets financés du système, de sorte que l'Orientation ait connaissance de la direction prise par ce dernier à travers la réalisation de ces projets. On comprend ici que la définition de la notion d'impact est au cœur de l'Évaluation.

Arrêter une définition formelle définitive de la notion d'impact est une tâche très complexe qui dépasse les ambitions du présent article. Pour les besoins de notre argument ici, il sera suffisant de délimiter le périmètre de la définition de la notion d'impact comme le proposent par exemple (Meinard et al., 2015) en distinguant réalisations, résultats et impacts :

- Les réalisations correspondent à ce qui est fait concrètement : par exemple, un projet de recherche est déployé sur le développement d'un nouveau vaccin ;

- Les résultats sont ce à quoi on aboutit en conséquence directe des réalisations : par exemple, des publications, des brevets et un vaccin commercialisé ;

- Les impacts désignent les conséquences des réalisations replacées dans leur contexte, incluant l'intervention de tous les autres acteurs susceptibles d'utiliser les résultats, de développer des alternatives, etc. : dans notre exemple, l'impact du projet de recherche pourrait être une diminution de la mortalité infantile ou une meilleure qualité de vie pour les personnes âgées.

Dans cette vision de la notion d'impact, il est clair que l'impact est une notion relative et non absolue, en ce sens que les éléments qui vont compter dans l'appréhension de l'impact vont dépendre de la politique dans le cadre de laquelle on cherche à appréhender l'impact. Par exemple, dans le cadre d'une politique de création d'emplois, le nombre d'emplois créés sera un élément majeur à prendre en compte dans l'appréhension de l'impact, alors qu'il ne le sera pas forcément pour une politique de rattrapage technologique. Pour chaque politique, le périmètre précis de la notion l'impact est en ce sens une composante de cette politique. C'est cette diversité des périmètres possibles de la notion d'impact, reflétant directement la diversité des politiques possibles, qui limite l'exercice de formalisation d'une définition générale et définitive de la notion d'impact d'un projet.

Pour la suite de cet article, il ne sera pas nécessaire d'aller plus loin dans la définition. Nous postulerons qu'un projet de recherche dispose de nombreux 
impacts et que l'Évaluation est capable de réaliser une agrégation de tous ces éléments selon une méthode que l'Orientation aura intégrée dans sa politique.

Dans ce qui suit, nous précisons les différences entre évaluation de la recherche et évaluation du système. En d'autres termes, nous présentons en quoi l'Évaluation telle que nous l'avons définie précédemment diffère de l'évaluation de la recherche telle qu'elle est généralement entendue par la majeure partie de la communauté scientifique. Nous discutons également de la notion d'indicateur, qui est une composante essentielle de toute évaluation et qui, concernant l'évaluation du système, représente une caractéristique fondamentale de sa dimension politique.

\section{L'évaluation de la recherche et du système : un problème de périmètre et de méthode}

Dans sa conception classique, l'évaluation de la recherche évalue la qualité de celle-ci. Son objectif est donc de répondre aux questions du type «Le projet est-il bon ou mauvais? Les travaux de ce chercheur sont-ils meilleurs que ceux d'un autre chercheur? Quel est le meilleur des centres de recherche français? ». De ce fait, le périmètre d'évaluation est limité à la fonction Recherche et s'inscrit dans un contexte d'intérêt particulier qui correspond à une unité bien définie (projet, chercheur, centre de recherche...). L'évaluateur s'intéresse donc aux qualités intrinsèques de l'unité évaluée sans réfléchir à son insertion et à son impact dans un système plus global. Un projet moyen d'un point de vue scientifique peut en effet avoir un impact positif important sur l'ensemble du système, s'il crée beaucoup d'emplois par exemple.

Dans ce cadre, alors que l'évaluation de la recherche dispose d'un périmètre limité à l'unité évaluée, l'évaluation du système s'intéresse à ce dernier dans sa globalité et œuvre à l'aiguiller dans une direction donnée (par l'Orientation). Ainsi, la fonction de l'Évaluation n'est pas de déterminer la qualité d'une recherche mais plutôt d'informer l'Orientation sur l'atteinte des objectifs politiques qu'elle a fixés et, dans le cas contraire, sur la direction prise par le système. En outre, bien que l'information portée par l'Évaluation puisse être d'ordre scientifique, sa valeur principale pour l'Orientation réside dans sa capacité à analyser l'impact global des projets de recherche financés. L'analyse des retombées de la recherche prend ici toute son utilité dans le sens où l'Orientation peut ainsi, en théorie, faire le lien entre l'investissement dans la recherche et son impact. Dans ce contexte, l'évaluation de la recherche est une composante de l'évaluation du système. En effet, là où l'évaluation de la recherche donne une vision microscopique de projet, l'évaluation donne une vision macroscopique du système. 
Enfin, alors que l'évaluation de la recherche est en quête de critères absolus ${ }^{7}$, l'évaluation du système dispose de critères relatifs fixés au préalable par l'Orientation et intégrés dans sa politique. De ce fait, il n'existe pas de bon ou de mauvais projets lorsque l'on évalue le système, mais plutôt : d'un côté, les projets dont l'impact attendu s'inscrit dans le cadre fixé par l'Orientation, et de l'autre, les projets restants. Lévaluation du système est donc radicalement différente de l'évaluation de la recherche aussi bien au niveau de son périmètre d'étude que de ses fondements méthodologiques.

\section{Des critères d'Évaluation au service de l'Orientation}

Un élément important dans la construction d'une méthodologie d'évaluation est l'utilisation d'indicateurs (généralement de performance) capables de traduire la réalité observée empiriquement en une représentation synthétique et fidèle de cette dernière.

La question de la construction des indicateurs pose ainsi souvent problème aux chercheurs en aide à la décision (voir par exemple Marchant et al., 2003 et Bouyssou et al., 2000) qui s'interrogent en particulier sur leur crédibilité. En effet, l'intérêt majeur des indicateurs se concentre dans l'image synthétique et compréhensible qu'ils donnent de la réalité. Le problème « [e]st que, de même qu'il n'existe pas de procédure universelle pour agréger l'information, il n'existe pas de méthode qui ait une validité générale pour évaluer un phénomène » (Tsoukias, 2004, p. 1).

Chaque indicateur, chaque méthode d'évaluation traduit ainsi une stratégie, des priorités et des politiques qui sont établies - généralement sans le savoir - avant l'évaluation elle-même.

Prenons quelques exemples. L'illustration la plus évidente correspond à la moyenne arithmétique des notes d'un élève. L'utilisation de cette méthode admet que l'on accepte que l'étudiant puisse compenser ses mauvaises notes avec ses bonnes notes et donc qu'il se concentre sur les matières où il a des facilités au détriment des autres. En revanche, si on utilise le minimum des notes au lieu de la moyenne (comme dans l'exemple documenté par Marchant et al. (2003) et Bouyssou et al. (2000), on n'accepte plus la compensation et l'élève aura tendance à se concentrer sur les matières où il est le plus en difficultés.

Derrière chaque méthode d'évaluation ou chaque indicateur on peut ainsi retrouver une stratégie (plus ou moins consciente) qui induit des

7. L'évaluation de la recherche est la source d'importants débats méthodologiques au sein de la communauté scientifique (Zaccai et al., 2016 ; Marjanovic et al., 2009). Ces derniers s'articulant principalement autour de l'utilisation de méthodes quantitatives (Andras, 2011), avec en tête le recours presque exclusif à la bibliométrie pour la recherche de critères d'évaluation absolus (Gingras, 2014 ; Harzing, Adler, 2009; Gläser, Laudel, 2005). 
comportements différents. Dans le cas de la moyenne, les étudiants auront tendance à se spécialiser dans les matières où ils ont le plus de facilité, alors que l'utilisation du minimum a pour conséquence une uniformisation du niveau vers le bas.

Au travers de plusieurs exemples, (Marchant et al., 2003) mettent en évidence des problèmes de normalisation, de construction d'échelles, de compensation, de monotonie et de signifiance propres aux indicateurs. En outre, avec l'apport de la théorie du mesurage (voir notamment Krantz et al., 1971 ; Massonie, 1988 ; Martel, Roy, 2002 ; Roberts, 1984 ), nous savons qu'il est bien souvent impossible de s'assurer que la construction d'un indicateur ne conduit pas à des situations paradoxales où l'information portée par l'indicateur serait en totale contradiction avec l'observation de la réalité. De ce fait, un indicateur n'aide pas à découvrir la vérité mais au contraire il la construit (Desrosieres, 1995). Les indicateurs aident ainsi à mettre en place une « Politique » en incitant les « Observés » à s'adapter à l'indicateur.

La fonction Évaluation doit ainsi être perçue dans un contexte qui ne se limite pas à la simple évaluation du système, mais bien comme une composante essentielle de la définition de la politique nationale de recherche et d'innovation. Dans ce contexte, une attention toute particulière doit être portée sur la construction des indicateurs de l'évaluation pour ne pas risquer d'induire des comportements contre-productifs.

\section{Organisation hiérarchique et fonctionnelle}

La première étape de cette construction consiste à reprendre les quatre fonctions préalablement définies et à préciser leur organisation hiérarchique et fonctionnelle. Nous allons donc scinder le système en deux parties avec, d'un côté, des éléments quantitatifs, et de l'autre, des éléments qualitatifs (voir Figure 4).

L'Orientation $^{8}$ se divise en politique quantitative et politique qualitative. La première correspond au budget du financement de la recherche en France et la seconde correspond aux orientations stratégiques que doit viser la recherche. Dans l'Hexagone, cette orientation est définie par la stratégie nationale de la recherche (SNR). La SNR, publiée en 2014 (MESR, 2014a) à la suite d'un travail collaboratif (MESR, 2014b) qui a réuni pendant plus de deux ans des centaines de chercheurs, est constituée de 10 défis que doit relever la recherche française. Chacun de ces défis est composé de plusieurs orientations dont l'ambition est de préciser les axes de recherche prioritaires.

8. Notons que, dans cette représentation, nous avons fait le choix de ne pas représenter l'influence très importante de l'Europe. Nous avons considéré que cette dernière était déjà intégrée dans les choix réalisés par l'Orientation. 
Figure 4 - Organisation du système français du financement de la recherche

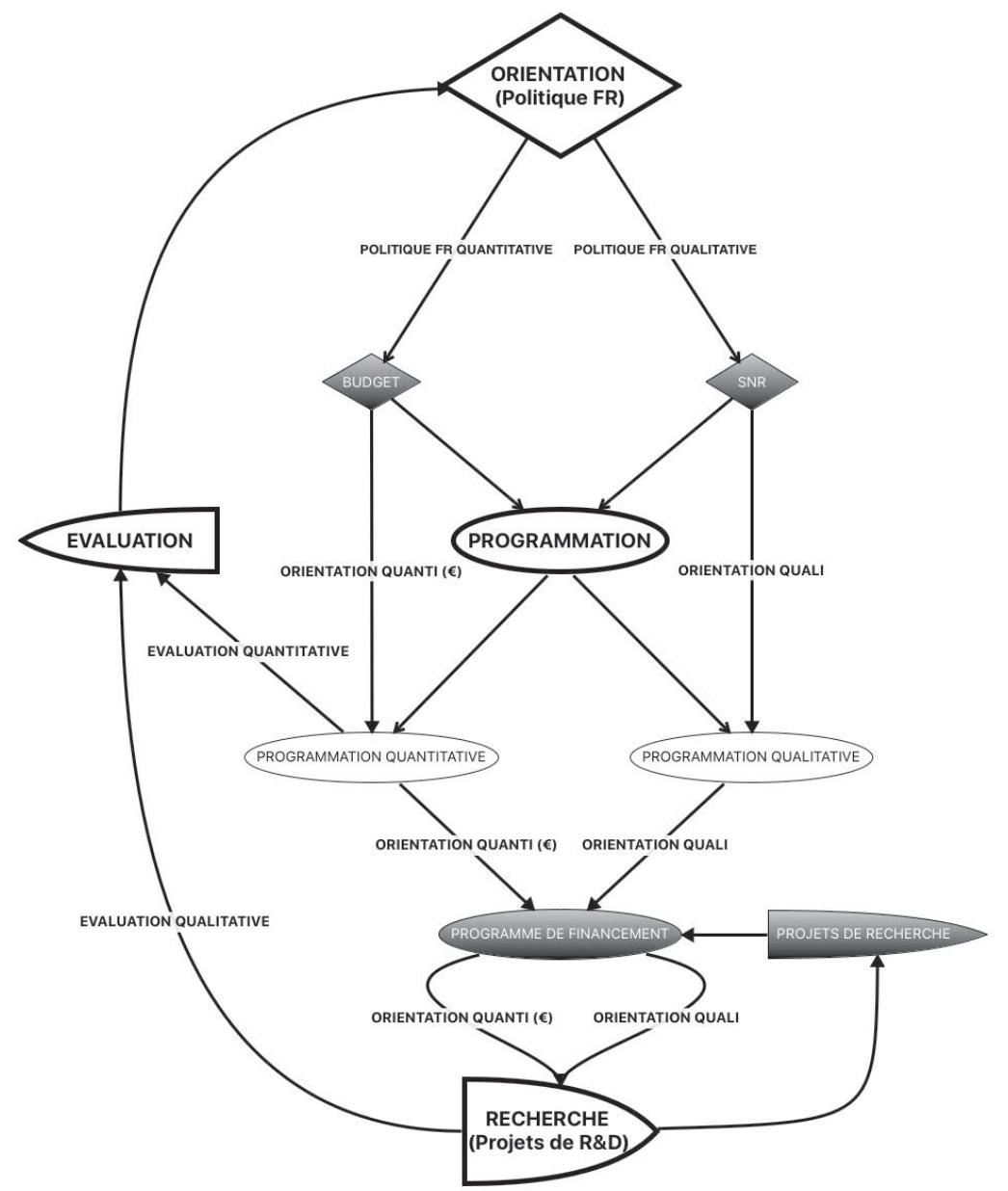

Dans ce même document, et à la suite des défis et orientations proposés par l'Orientation, la Programmation traduit ces ambitions en cinq programmes qui sont eux-mêmes divisés en actions, de sorte que la SNR puisse faire le lien entre l'Orientation qualitative du système et sa Programmation qualitative. La SNR tient donc une place très importante dans le système pour comprendre ses orientations stratégiques.

De même que l'Orientation, la Programmation se divise également en programmation qualitative et quantitative. La première, comme nous venons de le voir, est constituée principalement de la SNR. La seconde reprend les éléments budgétaires de la MIRES (programmes budgétaires et actions budgétaires). 
Les éléments qualitatifs et quantitatifs de l'Orientation traduits par la Programmation se retrouvent dans un programme de financement qui hérite de l'ensemble de cette information. Ce dernier peut ainsi financer, parmi les projets proposés par la Recherche, ceux qui se rapprochent le plus des thématiques de recherche visées sous la contrainte budgétaire imposée préalablement.

Pour finir, l'Évaluation récupère l'information relative aux projets financés en évaluant, dans le cadre d'une politique donnée par l'Orientation, les éléments quantitatifs fournis par les indicateurs de la programmation budgétaire (exemple : nombre de créations d'emplois de chercheurs) et les éléments qualitatifs fournis par la nature des projets financés (exemple : création d'une nouvelle technologie).

\section{Évaluation quantitative du SNFRI français}

L'évaluation quantitative du système français s'inscrit ainsi en partie dans le cadre d'une évaluation budgétaire. En effet, depuis l'instauration définitive de la LOLF en 2006, la MIRES est organisée en programmes et actions budgétaires. Ces derniers viennent généralement alimenter des fonds budgétaires qui financent enfin un programme de financement. Dans ce contexte, chaque action budgétaire s'articule autour d'objectifs clairs évalués par des indicateurs pour l'essentiel quantitatifs. L'OST (Observatoire des sciences et techniques) est ainsi en charge de l'évaluation quantitative du SNFRI, voir Figure 5.

Figure 5 - Évaluation quantitative du SNFRI

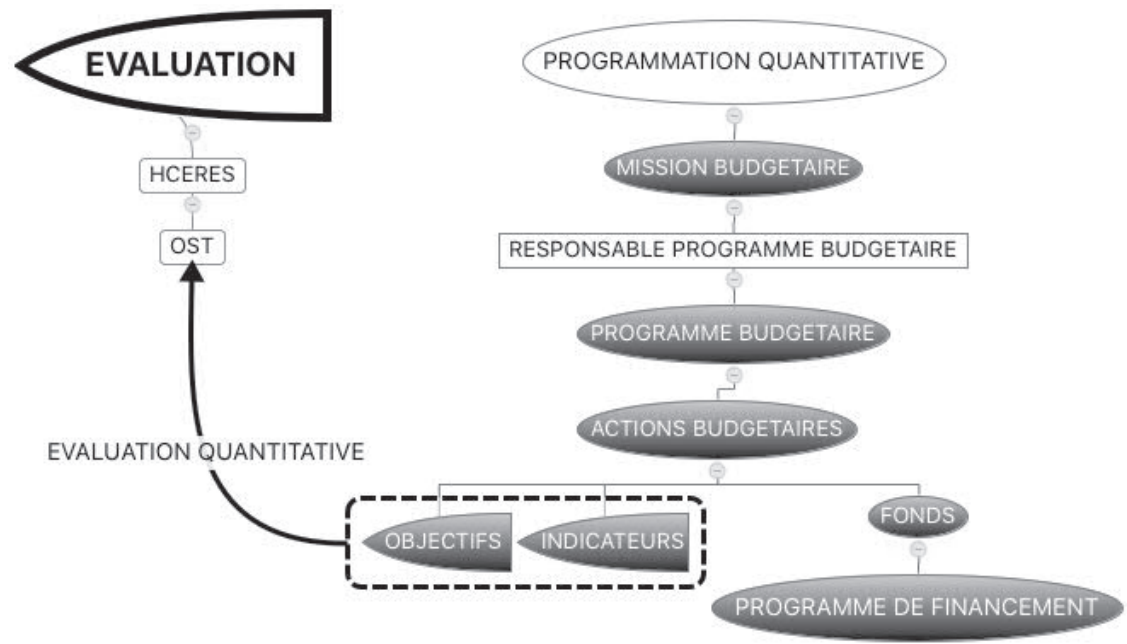




\section{Évaluation qualitative du SNFRI français}

En parallèle, l'évaluation qualitative du SNFRI est réalisée à deux niveaux du système (voir Figure 6).

Pour commencer, nous devons introduire deux niveaux d'opérateurs du système qui se différencient par leur rôle dans ce dernier :

- Opérateurs de niveau 1 qui financent les projets de recherche

- Opérateurs de niveau 2 qui exécutent des projets de recherche

Les opérateurs de niveau 1 appartiennent ainsi à la DNRD tandis que les opérateurs de niveau 2 sont comptabilisés dans la DIRD.

Cette distinction entre les opérateurs n'est pas exclusive. Un même opérateur peut à la fois être de $1^{\text {er }}$ et de $2^{\text {nd }}$ niveau. Les très grands laboratoires publics de recherche peuvent par exemple être amenés à financer des projets extérieurs sans avoir un rôle actif dans ces travaux de recherche. En outre, il existe d'autres opérateurs qui se limitent à l'instruction de la demande de financement pour le compte d'un opérateur de niveau 1. Enfin, depuis plusieurs années, on voit se multiplier des structures de coopération chargées de faire émerger de nouveaux projets, sans les financer ou y prendre part. Pour une meilleure lisibilité du système, les opérateurs chargés de l'instruction, de par leur grande proximité avec les opérateurs de niveau 1, sont rangés dans cette même catégorie. En revanche, pour les structures de coopération, nous créons un niveau 1,5 où nous réunissons tous les opérateurs de ce type.

La frontière entre $1^{\text {er }}$ et $2^{\text {nd }}$ niveau est donc perméable. En revanche, la question du rôle de chaque opérateur reste primordiale pour comprendre sa place dans le SNFRI. Ce dernier est ainsi fonction du programme de financement étudié (voir Figure 6). Selon le programme de financement, un même opérateur peut donc être de niveau 1 ou 2. En revanche, pour un programme donné, nous disposons dans la plupart des cas d'une information claire (généralement dans le cahier des charges ou le texte de l'appel à projets du programme) sur le rôle de chaque opérateur.

Dans ce contexte, le haut conseil de l'évaluation de la recherche et de l'enseignement supérieur $\left(\mathrm{HCERES}^{9}\right)$ peut évaluer tous les 5 ans chaque

9. L'objet de cet article n'est pas une critique du HCERES ou de l'AERES qui l'a précédé, mais nous pouvons quand même souligner quelques points de réflexion. En particulier, l'intérêt de telles évaluations réside dans le fait que celles-ci soient reconnues et acceptées. En l'espèce, les très nombreuses évolutions méthodologiques réalisées par l'AERES (voir notamment Glaudes, 2014) montrent une relative fébrilité dans l'acceptabilité de ces évaluations. Nous pouvons également ajouter les difficultés de l'évaluation de la production scientifique, liée aux sciences humaines et sociales comparativement aux sciences dites exactes. Enfin, nous pouvons nous étonner de la mission confiée au HCERES : «L'évaluation a posteriori des programmes d'investissement et des structures de droit privé recevant des fonds publics destinés à la recherche ou à l'enseignement supérieur » (http://www.hceres.fr/PRESENTATION/Missions). En effet, à notre connaissance, aucune publication à ce sujet n'a été pour l'instant présentée par cette institution. Nous pouvons cependant souligner l'effort 
opérateur selon un protocole largement qualitatif établi à l'avance et connu de tous (voir Figure 6).

Enfin, en Figure 6, nous avons représenté le trajet de l'argent qui passe par la DNRD, puis par le programme de financement (en suivant le chemin présenté précédemment) pour finalement financer un opérateur de niveau 2 et réaliser un projet de recherche qu'il a préalablement présenté à un programme de financement.

Figure 6 - Évaluation qualitative du SNFRI

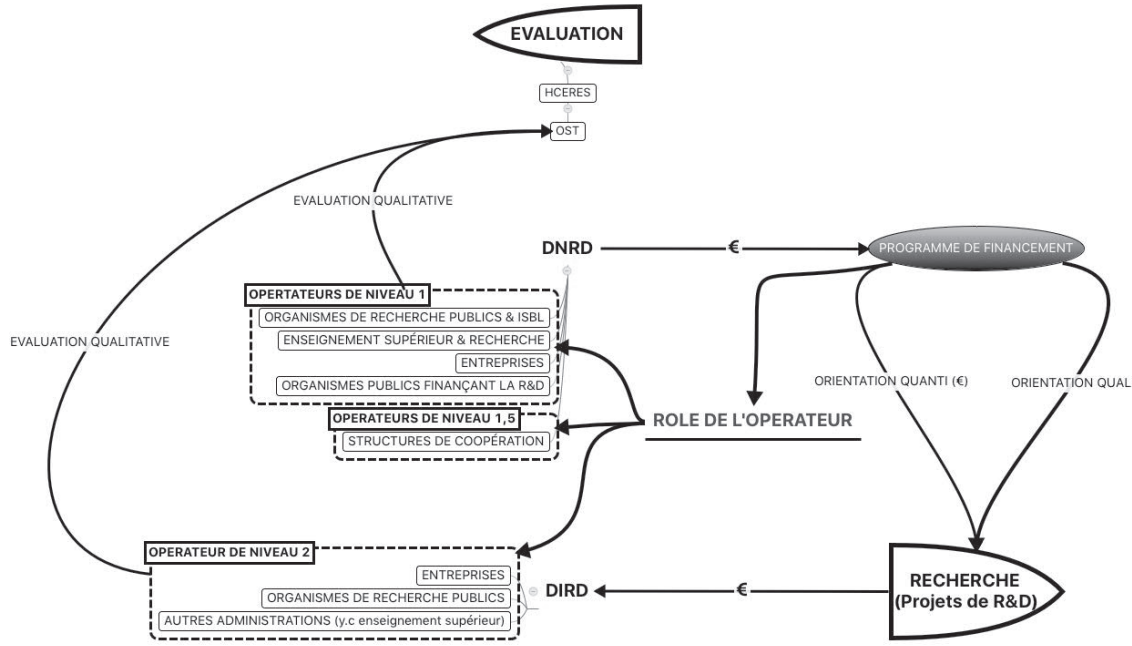

\section{Processus de décision du financement d'un projet}

Nous avons représenté en Figure 7 le processus de décision de financement d'un projet ${ }^{10}$. L'objectif ici n'est pas de détailler chaque processus au niveau du projet, mais plutôt de comprendre la mécanique générale commune à l'ensemble des programmes de financement. Ainsi, en Figure 7, nous représentons la décision de financement comme faisant le pont entre le programme de financement, représenté par les opérateurs de niveau 1 qui instruisent une demande (en finançant des projets), et la recherche représentée par les opérateurs de niveau 2 qui formulent une demande (en présentant des projets). La décision de financer un projet est ainsi un processus de décision multicritère dont le poids et l'évaluation des critères ainsi que la méthode

du gouvernement d'avoir inscrit dans les statuts du HCERES cette mission qui, nous l'espérons, permettra, comme nous essayons modestement de le faire, de mieux comprendre certaines décisions de financement.

10. Notons que ce dernier est généralement spécifique à chaque programme de financement. 
d'agrégation dépendent du programme de financement, qui lui-même dépend de la Programmation qui l'a créé en traduisant une orientation politique. De même, le budget joue un rôle important dans le nombre de projets financés en rajoutant une contrainte supplémentaire dans le processus de décision, voir notamment (Roy, 1985). Il est enfin intéressant de noter que cette décision alimente également, à travers les caractéristiques des projets financés, l'évaluation quantitative du système.

Figure 7 - Processus de décision de financement d'un projet

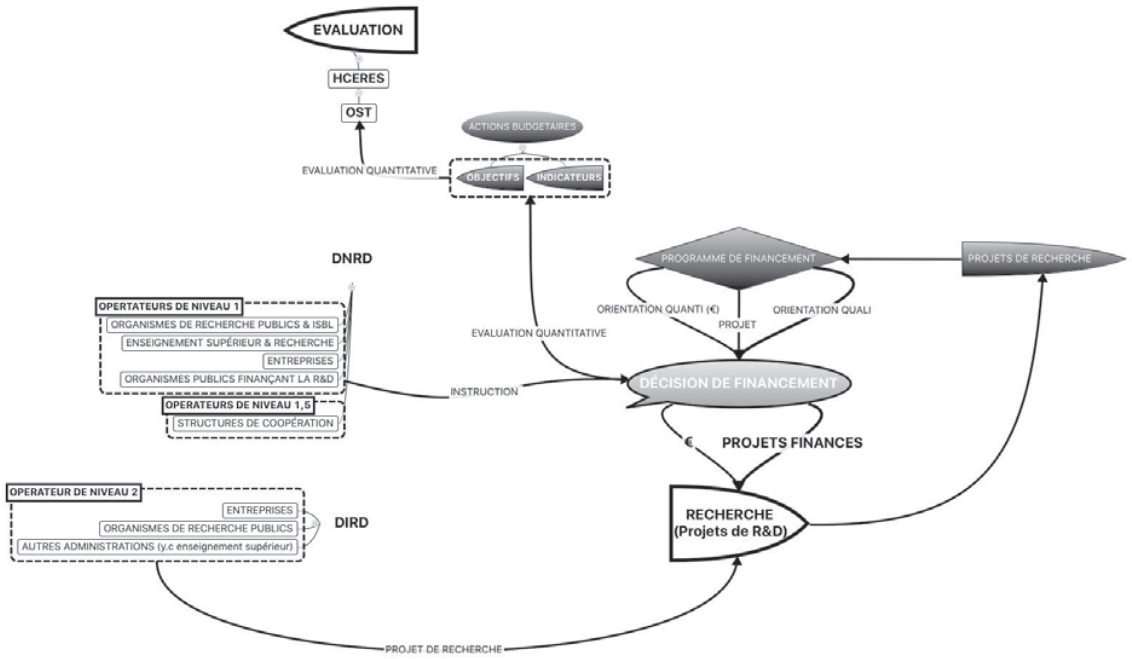

\section{Processus détaillé du financement d'un projet dans le cadre du SNFRI français}

Pour finir, nous avons réuni en Figure 8 les figures 5, 6 et 7 pour construire la carte détaillée du processus de financement d'un projet de recherche dans le cadre du SNFRI français ${ }^{11}$.

11. Le lecteur qui souhaite retracer le trajet de l'argent pourra noter qu'il n'existe pas de lien graphique entre le budget et la DNRD, cette dernière pointant directement sur le programme de financement sans aucune source. Il est évident que la DNRD est alimentée par le budget. En outre, le parcours des fonds suit bien le schéma présenté précédemment (mission budgétaire, programme budgétaire, actions, fonds). Le choix de ne pas représenter ce parcours a cependant été fait pour ne pas alourdir la représentation du SNFRI. 


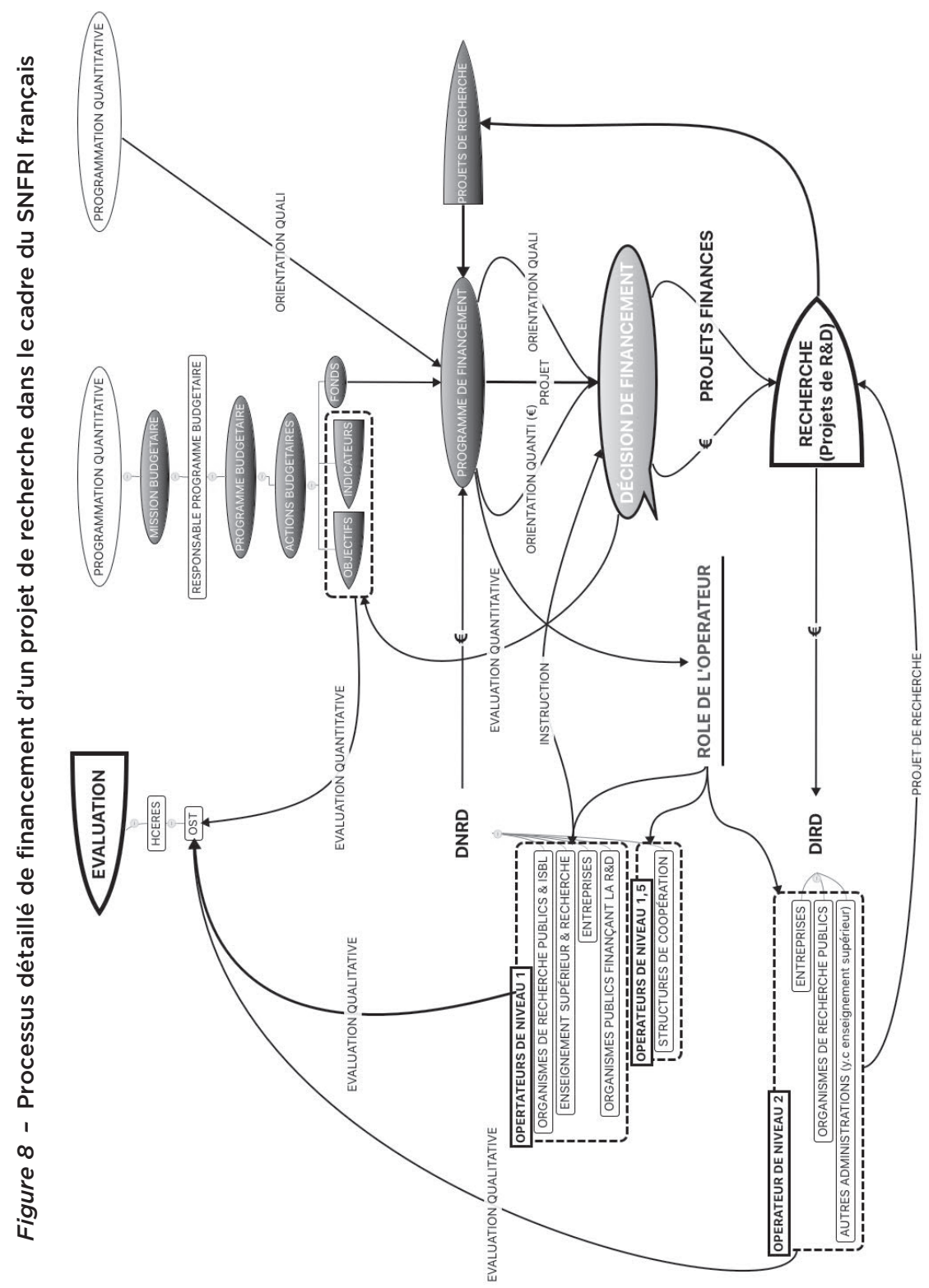


Pour illustrer cette dernière carte et surtout valider la pertinence de nos quatre fonctions pour expliquer la décision de financer un projet, prenons l'exemple (fictif) d'un programme qui vise à financer le développement d'une voiture volante. Nous considérerons ici comme acquis que l'Orientation a fait figurer dans ses priorités le développement des voitures volantes et y a dédié un budget. Dans ce cadre, il existe une action budgétaire qui vise à financer des projets permettant le développement de la voiture volante, et un fond «Voiture volante " a même été créé à cet effet. Associée à l'action budgétaire, l'Orientation a défini un certain nombre d'objectifs et d'indicateurs dont elle a laissé le soin à l'HCERES et à l'OST de les évaluer. Forte de cette politique «Voiture volante », la Programmation a construit un programme dédié au financement de projets devant permettre le développement de la voiture volante. Ce programme hérite ainsi d'un budget et d'une orientation scientifique et/ou technologique préalablement établie par l'Orientation. De même, ce programme dispose de règles de financement ainsi que d'une définition des rôles de chaque opérateur qui intervient dans le choix des projets à financer. Ces derniers étant évalués en fonction des objectifs et indicateurs inscrits dans l'action budgétaire, on peut considérer que le programme de financement hérite également de ces objectifs et indicateurs.

Les opérateurs de niveau 2 vont donc venir proposer des projets dans le cadre du programme de financement «Voiture volante » et des opérateurs de niveau 1 vont instruire cette demande pour ensuite décider ou non de les financer. Cette décision est établie en fonction de l'adéquation du projet aux attributs du programme de financement.

Les projets choisis seront ainsi financés dans le cadre de la DNRD et les dépenses réalisées dans le cadre de ce projet seront comptabilisées dans le cadre de la DIRD.

Enfin, les caractéristiques de l'ensemble des projets financés seront agrégées par l'Évaluation à travers les indicateurs et objectifs préalablement établis pour remonter à l'Orientation une vision la plus fidèle possible de l'atteinte ou non des objectifs qu'elle avait définis, cette évaluation pouvant servir à faire évoluer sa politique ou en construire une nouvelle.

La cartographie du système à travers les fonctions Orientations, Programmation, Recherche et Évaluation apporte ainsi des éléments objectifs permettant de comprendre les déterminants de la décision de financement d'un projet. Nous en déduisons que ces fonctions sont pertinentes. 


\section{Conclusion}

En partant du modèle de SNRI de Barré et de la théorie des systèmes d'innovation, nous avons proposé une carte du système français du financement de la recherche qui s'articule autour des trois fonctions de Barré (Orientation, Programmation et Recherche) auxquelles nous avons ajouté une fonction Évaluation. Cette carte a été construite dans le cadre d'une observation participante opportuniste dont la principale limite est le possible manque d'exhaustivité. Bien que nous n'ayons pas identifié de dispositif de financement qui ne s'intégrerait pas dans cette carte, le nombre de dispositifs est tel qu'il est impossible de garantir l'exhaustivité.

L'Évaluation a été construite pour informer l'Orientation de la direction prise par le système en agrégeant l'impact de tous les projets financés. Nous avons caractérisé l'impact d'un projet de recherche comme étant une notion relative à la politique dans laquelle elle s'inscrit. Dans notre modèle, pour simplifier la lecture du système, nous avons fait le choix de limiter l'Évaluation à la mesure de l'écart entre la direction souhaitée par l'Orientation et la direction réellement prise par le système. Notons cependant que, par nature, une politique publique de recherche peut être qualifiée de « multifonctionnelle ", en ce sens que, au-delà des objectifs qu'elle s'était fixés, elle peut influer directement et indirectement sur toute la société à travers de multiples progrès technologiques, sociaux, environnementaux, économiques, etc. Une Évaluation qui serait capable de prendre en compte cette « multifonctionnalité » pourrait servir de guide à l'Orientation, non plus seulement en mesurant l'écart entre les objectifs initialement fixés et les aboutissements réels, mais en suggérant de nouvelles directions, voire même en identifiant de nouveaux impacts positifs non-prévus lors de conception de la politique. Une telle Évaluation serait bien plus ambitieuse et, en un sens, ouverte, que la fonction Évaluation telle que nous l'avons définie. Elle ne se limiterait plus à analyser les impacts en fonction des objectifs initiaux de la politique publique dans laquelle elle s'inscrit : elle les analyserait au contraire également au prisme d'objectifs différents, que la politique aurait pu s'assigner. Une tentative de démarche de ce type est proposée par (Meinard et al., 2015), qui analysent une politique sur la base d'un cadre d'enjeux génériques construit ex-post. Lélaboration d'une méthodologie solide pour ce type de démarche est une frontière de recherche importante pour les prolongements de notre travail.

Au travers de notre carte, nous avons attesté empiriquement du fait que les quatre fonctions définies précédemment existent bien dans le système et permettent d'éclairer une décision de financement d'un projet. Cet article est ainsi la première étape d'un travail plus large dont l'objectif final est de 
construire un modèle de SNFRI capable de l'évaluer et d'appréhender sa dynamique.

Le modèle que nous souhaitons construire repose sur la construction d'une fonction projet (qui fait le lien entre les caractéristiques d'un projet de recherche et son impact global) et s'articule autour d'une organisation circulaire de l'information dont le principal moteur est l'évaluation du système.

Enfin, ce modèle reprendra le cadre de l'évaluation du système à travers un suivi d'indicateurs préalablement construits par l'Orientation et directement intégrés dans la politique.

\section{RÉFÉRENCES}

ABERCROMBIE, N., HILL, S., TURNER, B. S. (2000), Participant Observation, in Dictionary of Sociology, Thousand Oaks, California, Penguin Books, 256.

ADLER, P., ADLER, P. (1987), Membership Roles in Field Research, Sage Publications.

AMABLE, B. (2002), Les Systèmes d'innovation, in Mustar, P., Penan, H. (dir.), L'Encyclopédie de l'Innovation, Paris, Economica, 1-14.

ANDRAS, P. (2011), Research: Metrics, Quality, and Management Implications, Research Évaluation, 20(2), 90-106.

BARRE, R. (2007), Essai d'interprétation de l'évolution 2006-2007 du SFRI : la réforme à la croisée des chemins, La Recherche et l'Innovation en France - Rapport Futuris 2007, Paris, Odile Jacob.

BARRE, R. (2011), Programmation de la recherche : perspectives conceptuelles, institutionnelles et actuelles, Innovations, 36(3), 9-19.

BARRE, R. (2016), Une brève histoire du SFRI Un regard quantitatif (1963-2013), La Recherche et l'Innovation en France - Rapport Futuris 2015-2016, Paris, Odile Jacob.

BERGEK, A., JACOBSSON, S. (2003), The Emergence of a Growth Industry: A Comparative Analysis of the German, Dutch and Swedish Wind Turbine Industry, in Metcalfe, J. S., Cantner, U. (dir.), Change, Transformation and Development, PhysicaVerlag HD.

BERGEK, A., JACOBSSON, S., CARLSSON, B., LINDMARK S., RICKNE, A. (2008), Analyzing the Functional Dynamics of Technological Innovation Systems: A Scheme of Analysis, Research Policy, 37, 407-429.

BETA (1988), Etude sur les effets économiques des dépenses spatiales européennes, Agence Spatiale Européenne.

BOUYSSOU, D., MARCHANT, T., PIRLOT, M., PERNY P., TSOUKIAS, A., VINCKE, P. (2000), Évaluation and Decision Models: A Critical Perspective, International Series in Operations Research and Management Science, Kluwer Academic Publishers.

CARLSSON, B., JACOBSSON, S., HOLMÉNB M., RICKNE, A. (2002), Innovation Systems: Analytical and Methodological Issues, Research Policy, 31, 233-245.

CARLSSON, B., STANKIEWICZ, R. (1995), Technological Systems and Economic Performance: The Case of Factory Automation, in Carlsson, B. (eds), On the Nature, Function and Composition of Technological Systems, Kluwer Academic Publishers. 
CASADELLA, V., BENLAHCEN-TLEMCANI, M. (2006), De l'applicabilité du système national d'innovation dans les pays moins avancés, Innovations, 24(2), 59-90.

CNER (1994), Évaluation du programme spatial français, in CNER, Un autre regard sur la recherche, Comité National d'Évaluation de la Recherche.

COHENDET, P., LEBEAU, A. (1987), Choix stratégiques et grands programmes civils, Paris, Economica.

COUR DES COMPTES (2013), Le financement public de la recherche, un enjeu national.

CHESNAIS, F. (1993), The French National System of Innovation, in Nelson, R. (dir.), National Innovation Systems: A Comparative Analysis, Oxford University Press.

CRESPY, C., HERAUD, J.-A., PERRY, B. (2007), Multi-Level Governance, Regions and Science in France, Regional Studies, 41(8), 1069-1084.

DE SARDAN, J. P. O. (2001), L'enquête de terrain socio-anthropologique, Enquête, 8, 63-81.

DESROSIERES, A. (1995), Refléter ou instituer: L'invention des indicateurs statistiques, INSEE.

DIAZ, F. (2005), L'observation participante comme outil de compréhension du champ de la sécurité, Champ pénal [en ligne], 2, mis en ligne le 30 janvier 2005, consulté le 21 août 2018, http://journals.openedition.org/champpenal/79 ; DOI: 10.4000/champpenal.79

DODET, M., LAZAER, P., PAPON, P. (1998), La République a-t-elle besoin de Savants ?, Paris, PUF.

EDQUIST, C. (1997), Systems of Innovation, Technologies, Institutions and Organizations, Londres, Pinter.

EDQUIST, C. (2006), Systems of Innovation: Perspectives and Challenges, in Edquist, C., Gagerberg, J., Mowery, D. C. (eds), The Oxford Handbook of Innovation, Oxford University Press.

ERGAS, H. (1987), Does Technology Policy Matter, Center for European Policy Studies.

FREEMAN, C. (1987), Technology Policy and Economic Performance: Lessons from Japan, Londres, Pinter.

FREEMAN, C. (1995), The National System of Innovation in Historical Perspective, Cambridge Journal of Economics, 19(1), 5-24.

GILPIN, R. (1970), La Science et l'Etat en France, Paris, Gallimard.

GINGRAS, Y. (2014), Les dérives de l'évaluation de la recherche. Du bon usage de la bibliométrie, Paris, Raisons d'Agir.

GLÄSER, J., LAUDEL, G. (2005), Advantages and Dangers of "Remote" Peer Évaluation, Research Évaluation, 14(3), 186-198.

GLAUDES, P. (2014), L'évaluation de la production scientifique en France par l'agence d'évaluation de la recherche et de l'enseignement supérieur, Mélanges de la Casa de Velázquez, 44(2), 293-300.

GUILlAUME, H. (1998), Rapport de Mission sur la Technologie et l'Innovation, Paris, Documentation française.

HARZING, A. W., ADLER, N. (2009), When Knowledge Wins: Transcending the Sense and Nonsense of Academic Rankings, The Academy of Management Learning and Education, 8(1), 72-95. 
HEKKERT, M., SUURS, R., NEGRO, S., KUHLMANN, S., SMITS, R. (2007), Functions of Innovation Systems: A New Approach for Analysing Technological Change, Technological Forecasting and Social Change, 74, 413-432.

HEKKERT, M., NEGRO, S. (2009), Functions of Innovation Systems as a Framework to Understand Sustainable Technological Change: Empirical Evidence for Earlier Claims, Technological Forecasting and Social Change, 76, 584-594.

HERAUD, J.-A., LACHMANN, J. (2015), L'évolution du système de recherche et d'innovation : ce que révèle la problématique du financement dans le cas français, Innovations, 46(1), 9-32.

HUGHES, T. P. (1990), The Evolution of Large Technological Systems, in Bijker, W., Hughes, T. P., Pinch, T. J. (eds), The Social Construction of Technological Systems: New Directions in the Sociology and History of Technology, Chicago, The MIT Press.

HUGHES, E. C. (1996), Le regard sociologique : essais choisis, Paris, Edition de l'EHESS.

JACOBSSON, S., SANDEN, B., BANGENS, L. (2004), The Evolution of the German Technological System for Solar Cells, Technology Analysis and Strategic Management, 16(1), 3-30.

JOHNSON, A. (2001), Functions in Innovation System Approaches, Nelson and Winter Conference, 12-15 juin, Aalborg.

KRANTZ, D., LUCE, R., SUPPES, P., TVERSKY, A. (1971), Foundations of Measurement, Vol. 1: Additive and Polynomial Representations, New York, Academic Press.

LAREDO, P., MUSTAR, P. (2001), French Research and Innovation Policy: Two Decades of Transformation, in Research and Innovation Policies in the New Global Economy: An International Comparative Analysis, Cheltenham, Edward Elgar, 447-497.

LEBEAU, A. (1985), Eléments d'une stratégie dans le domaine spatial. Rapport pour le ministre de l'industrie et de la recherché, CPE Mimeo.

LETOURNEAU, A. (2008), La transdisciplinarité considérée en général et en sciences de l'environnement, [VertigO] La revue électronique en sciences de l'environnement, 8(2), 1-9.

LORIDANT, P. (1991), Rapport sur les orientations de la politique spatiale française et européenne, Office parlementaire d'évaluation des choix scientifiques et technologiques.

LUNDVALL, B. (1992), National Innovation System: Toward a Theory of Innovation and Interactive Learning, Londres, Pinter.

LUNDVALL, B., JOHNSON, B. (1994), The Learning Economy, Journal of Industry Studies, 2(1), 22-43.

LUNDVAlL, B., JOHNSON, B., ANDERSEN, E.S., DALUM, B. (2002), National Systems of Production, Innovation and Competence Building, Research Policy, 31, 213-231.

LUNDVALL, B. (2007), Innovation System Research Where It Came From and Where it Might Go, Fifth Globelics Conference, September 19-23, Saratov.

MALERBA, F. (1999), Sectoral Systems of Innovation and Production, DRUID Conference on National Systems of Innovation, 9-12 juin, Aalborg.

MALERBA, F. (2002), Sectoral Systems of Innovation and Production, Research Policy, $31,247-264$.

MARCHANT, T., PERNY, P., PIRLOT, M., TSOUKIAS, A., VINCKE, P. (2003), Les indicateurs en perspectives, working paper. 
MARCHI, G. D., LUCERTINI, G., TSOUKIÀS, A. (2014), From Evidence-Based Making to Policy Analytics, Annals of Operations Research, 236, 15-38.

MARJANOVIC, S., HANNEY, S., WOODING, S. (2009), A Historical Reflection on Research Évaluation Studies, Their Recurrent Themes and Challenges, Rand EuropeTechnical Report.

MARTEL, J. M., ROY, B. (2002), Analyse de signifiance de diverses procédures d'agrégation multicritère, Cahier du LAMSADE, 199.

MASSONIE, J.-P. (1988), Introduction à la théorie de la mesure, Histoire $\mathcal{E}$ Mesure, 3, 7-18.

MEINARD, Y., CALENDOT, H., MARTIN, M. (2015), Évaluation et redéfinition de la politique départementale en faveur des milieux aquatiques (PADMA), Département de la Gironde.

MINISTERE DE L'ENSEIGNEMENT SUPERIEUR ET DE LA RECHERCHE (MESR) (2014a), Stratégie nationale de recherche - France Europe 2020, Paris.

MINISTERE DE L'ENSEIGNEMENT SUPERIEUR ET DE LA RECHERCHE (MESR) (2014b), Stratégie nationale de recherche - rapport de propositions et avis du conseil stratégique de la recherche, Paris.

MUSTAR, P., LAREDO, P. (2002), Innovation and Research Policy in France (1980-2000) or The Disappearance of the Colbertist State, Research Policy, 31, 55-72.

NEGRO, S. O., HEKKERT, M. P., SMITS, R. E. (2007), Explaining the Failure of the Dutch Innovation System for Biomass Digestion-a Functional Analysis, Energy Policy, 35, 925-938.

NELSON, R. (1993), National Innovation Systems: A Comparative Analysis, Oxford, Oxford University Press.

OECD (2002), Manuel de Frascati 2002 : Méthode type proposée pour les enquêtes sur la recherche et le développement expérimental.

PAILLOTIN, G. (1993), Recherche et Innovation : le temps des réseaux, Paris, Documentation française.

PLATT, J. (1983), The Development of the 'Participant Observation' Method in Sociology: Origin Myth and History, Journal of the History of the Behavioral Sciences, 19(4), 379-393.

RICKNE, A. (2001), Assessing the Functionality of an Innovation System, Nelson and Winter Conference, 12-15 juin, Aalborg.

ROBERTS, F. S. (1984), Measurement Theory with Applications to Decision Making, Utility and the Social Sciences, 7, Cambridge University Press.

ROY, B. (1985), Méthodologie multicritère d'aide à la décision, Paris, Economica.

SALOMON, J.-J. (1986), Le gaulois, le cow-boy, et le samouraï : la politique française de la technologie, Paris, Economica - Centre de Prospective et d'Évaluation.

SCHNEIDER, D. K. (1996), Modélisation de la démarche du décideur politique dans la perspective de l'intelligence artificielle, Thèse de Doctorat, Faculté des sciences économiques et sociales de l'Université de Genève.

SOULE, B. (2007), Observation participante ou participation observante ? usages et justifications de la notion de participation observante en sciences sociales, Recherches qualitatives, 27(1), 127-140. 
TENIERE-BUCHOT, P.-F. (1982), Évaluation de trois programmes de développement technologique : Electronucléaire, Aéronautique, Recherche spatiale, IDRH-CPE.

TSOUKIAS, A. (2004), Les enjeux de l'évaluation, Vie de la recherche Scientifique, 357, 32-33.

WEISENBURGER, E. (2014), L'état de l'Enseignement supérieur et de la Recherche en France, Ministère de la recherche et de l'enseignement supérieur, Paris.

ZACCAI, E., TIMMERMANS, B., HUDON, M., CLERBAUX, B., LECLERQ, B., BERSINI, H. (2016), L'évaluation de la recherche en question(s), Penser la science, Académie royale des Sciences, des Lettres et des Beaux-Arts de Belgique.

\section{Annexe: Le système français de recherche et d'innovation (sfri) de 1945 a nos jours}

Pour bien comprendre le fonctionnement du SFRI actuel, il est important de revenir sur ses racines historiques et en particulier ses différentes constructions et évolutions de la sortie de la Seconde Guerre mondiale à nos jours. Ce point de départ de notre analyse correspond à un moment critique pour la France qui, à la fin de la guerre, a dû faire face à un retard considérable tant sur le plan scientifique qu'économique. Pour expliquer les différentes évolutions de ce système, nous utiliserons Heraud et Lachmann (2015) et Mustar et Larédo (2002) qui citent Chesnais (1993), lui-même s'étant inspiré de Salomon (1986) et Gilpin (1970) pour décrire deux périodes caractérisées par la place de l'Etat dans la recherche française.

L'analyse des 50 dernières années d'évolution du SFRI montre ainsi l'existence de deux cycles complets de réformes, caractérisés chacun par des lois de changement structurel : les lois de 1982-1984 et les lois de 2006-2007.

\section{De 1945 à 1980, un SFRI colbertiste}

À la sortie de la guerre, le SFRI s'est construit dans une dynamique de rattrapage technologique et scientifique impulsée par le Général De Gaulle et son commissariat général au plan caractérisé par un État centralisé que les auteurs qualifient de modèle Colbertiste (pour une étude approfondie, on peut se référer à Barré (2016), Heraud et Lachmann (2015), Mustar et Larédo (2002), Larédo et Mustar (2001), Chesnais, (1993), Gilpin (1970) et Salomon (1986)).

Ce modèle est défini par :

- Le centralisme du CNRS chargé de l'essentiel de la recherche fondamentale en France.

- La scission entre le CNRS chargé de la recherche et l'Université responsable de la formation des étudiants. Les jeunes sont de ce fait trop 
éloignés de la recherche avec de surcroît le système unique des grandes écoles qui ne réalisent pas de recherche mais captent l'essentiel des élites.

- La création de « champions nationaux » au sein de grands programmes de recherche thématiques qui captent l'essentiel du financement et qui ont été créés pour répondre à des objectifs d'indépendance stratégique.

- La multiplication de laboratoires publics de recherche appliquée ((Mustar et Larédo, 2002, parlent de «Mission Oriented») spécialisés dans un domaine (agriculture $=$ INRA, énergie et défense $=$ CEA, aérospatial et défense $=$ ONERA, etc.) qui travaillent en lien avec l'industrie et qui peuvent d'une certaine manière rester très éloignés de la recherche fondamentale.

Le SFRI Colbertiste d'après-guerre a été essentiel pour la recherche française de cette époque qui a rattrapé son retard scientifique avec de belles réussites technologiques telles que le TGV, le Concorde ou l'industrie nucléaire par exemple. Ce centralisme a permis notamment grâce aux grands programmes thématiques de faire avancer dans une même direction l'ensemble des acteurs d'un même domaine de recherche. En outre, jusqu'au milieu des années 1980, le SFRI était un élément de compétitivité nationale, la loi sur la recherche de 1982 ne fait en ce sens que confirmer le modèle en l'institutionnalisant.

Mais le développement de nouvelles technologies, liées notamment aux sciences de la vie et au numérique, devenues plus intéressantes économiquement que les applications de la physique (comme le nucléaire), permettent l'émergence de nouveaux acteurs privés, tout en imposant des collaborations scientifiques entre plusieurs domaines de recherche restés jusqu'à présent très cloisonnés. A cela, s'ajoutent la contestation du nucléaire avec l'apparition des premiers mouvements écologistes, la multiplication des mouvements sociaux au sein des chercheurs (dégradation des conditions et travail et du statut des chercheurs) ainsi qu'une revue des priorités budgétaires reléguant la recherche au second plan. Les années 80 marquent le début de la fin du modèle Colbertiste français et la fin de la première séquence politique.

\section{De 1980 à 2000, la fin du colbertisme}

Pour Mustar et Larédo (2002), le modèle français centralisé ou Colbertiste évolue à partir des années 1980 avec :

- La fin des grands programmes nationaux au profit de nombreuses privatisations (pour une évaluation des programmes : Ténière-Buchot, 1982 ; Lebeau, 1985 ; Cohendet, Lebeau, 1987 ; Loridant, 1991 ; C.N.E.R, 1994 ; Beta, 1988). 
- La création des UMR (unités mixtes de recherche) qui permettent une convergence du CNRS et de l'Université.

- La convergence des laboratoires publics de recherche appliquée «Mission Oriented » et de la recherche fondamentale. Ceci se caractérise avec notamment la création des EPST (établissements publics à caractère scientifique et technologique) - en complément des EPIC (établissements publics à caractère industriel et commercial) - qui alignent les modalités de recrutement et la gestion de carrière de ces laboratoires avec le CNRS et des incitations financières à la recherche collaborative.

- L'augmentation de la part des PME dans le SFRI permise par le déploiement de logique de financement «bottom-up» (création du crédit d'impôt recherche, de l'ANVAR et du PCRD).

- L'apparition de nouveaux acteurs publics dans le SFRI (pour une étude approfondie voir Paillotin, 1993 ; Dodet et al., 1998 ; Guillaume, 1998) avec notamment l'Europe (création du PCRD en 1984) et les régions (loi de décentralisation de 1982).

A partir des années 1980, on assiste ainsi à une évolution de la philosophie du SFRI avec en particulier, la découverte des PME par les pouvoirs publics, l'émergence de nouveaux acteurs et une incitation à la collaboration scientifique. Les différentes évolutions de cette époque correspondent à une tendance de fond qui vise à se rapprocher de ce que Ergas (1987) qualifie de modèle «Diffusion Oriented» (améliorer les capacités technologiques générales des entreprises) tout en s'éloignant du modèle «Mission Oriented » ( $R \& D$ pilotée par des grands programmes nationaux dirigés vers un but clairement défini).

Ainsi à partir des années 1990, on substitue aux grands programmes des problèmes de société (environnement, santé publique, urbanisme...) qui dépassent les frontières. On voit alors apparaitre des politiques supranationales avec notamment le PCRD. De même, les régions prennent une part toujours plus importante dans certaines thématiques de recherche. Pour qualifier ces évolutions, Crespy et al. (2007) explique que le SFRI se dirige vers une gouvernance multi-niveaux de la recherche. En outre, de nouveaux acteurs, et en particulier privés, émergent sur certaines thématiques de recherche tant et si bien que le ministère de la recherche (via l'Université, le CNRS, ou d'autres organismes publics) n'est plus le premier financeur de la recherche sur ces thématiques (exemple de la recherche sur les maladies rares avec le Généthon).

Pour Heraud et Lachmann (2015) le SFRI à partir des années 1990 devient donc non-seulement multi-niveaux, mais également multi-acteurs. 


\section{Les lois de recherche de 2006 et 2013 vers le renouveau du SFRI}

La période commence avec l'entrée en vigueur totale de la loi organique relative aux lois de finance (LOLF) qui instaure un nouveau paradigme budgétaire s'articulant autour d'une logique de performance d'une part et de transparence d'autre part. Dès lors et pour la première fois, le budget dispose d'objectifs clairement indiqués et d'indicateurs chiffrés pour valider l'atteinte de ces objectifs. À cette réforme structurelle s'ajoute un renforcement de la gouvernance multi-acteurs et multi-niveaux avec la création de l'agence nationale pour la recherche (ANR) en 2005 ainsi que la loi de programme sur la recherche de 2006. Cette loi qui est la plus importante depuis 1982 est caractérisée notamment par :

- Un objectif budgétaire dédié à la recherche qui comprend un montant réservé au financement de la recherche sur projet.

- L'encouragement des coopérations scientifiques à travers la création de très nombreux regroupements (PRES, RTRA, CTRS, Label Carnot).

- La création d'une agence d'évaluation de la recherche et de l'enseignement supérieur (AERES) remplacée en 2013 par le haut conseil de l'évaluation de la recherche et de l'enseignement supérieur (HCERES).

Cette loi est par ailleurs globalement confirmée par la loi sur l'enseignement supérieur et la recherche (ESR) de 2013. Le financement sur projet, la coopération scientifique et l'évaluation des personnels, des unités de recherche et des établissements deviennent ainsi la norme. La coopération scientifique va, en outre, être soutenue à travers la création des pôles de compétitivité en 2005 ainsi que des IDEX en 2011. Enfin, la loi liberté et responsabilité des universités (LRU) de 2007 fait évoluer le statut des universités vers plus d'autonomie.

Du point de vue du financement, ces années sont marquées d'une part par la création des investissements d'avenir, et d'autre part, par l'essor du crédit d'impôt recherche qui voit son assiette considérablement augmenter pour devenir la principale source de financement de la recherche industrielle en France.

Cependant, d'après Barré (2016) : « Ces réformes ont souvent conduit à des superpositions de dispositifs générant complexité et incertitudes. Nombreux sont ceux qui considèrent que le système est devenu largement illisible tant pour ses propres acteurs que pour l'extérieur. » 